\title{
Development of a Plasma Source Using Atmospheric-Pressure Glow Discharge in Contact with Solution
}

\author{
HIRAI Kazuhiko ${ }^{1)}$, OKADA Takeru ${ }^{1)}$, KANEKO Toshiro ${ }^{1)}$, HATAKEYAMA Rikizo ${ }^{1)}$ \\ and YOSHIKI Hiroyuki ${ }^{2}$ \\ ${ }^{1)}$ Department of Electronic Engineering, Tohoku University, Sendai, 980-8579, Japan \\ ${ }^{2)}$ Tsuruoka National College of Technology, 104 Sawada, Inooka, Tsuruoka, Yamagata, 997-8511, Japan \\ (Received 22 April 2005 / Accepted 7 May 2005)
}

\begin{abstract}
A novel plasma source of atmospheric-pressure glow (APG) discharge in contact with solution is developed. Liquid paraffin consisting of hydrocarbons is used as solution for the purpose of the formation of carbon nanotubes at the gas-liquid interface, i.e., the boundary between glow discharge (gas-phase) and solution (liquid-phase) layers. We have succeeded in generating a stable APG discharge plasma in contact with liquid paraffin by using a capacitively coupled plasma method with mesh electrode.
\end{abstract}

\section{Keywords:}

atmospheric-pressure, glow discharge plasma, mesh electrode, solution, gas-liquid, interface

In recent years, atmospheric-pressure discharge plasmas, for which no large-scale vacuum system is required, have been actively studied in regard to generation, diagnostics and possible applications [1-3]. The atmospheric-pressure discharge plasmas afford new parameter regions, for example, (i) high density, (ii) minute scale, and (iii) non-equilibrium, which are different from the conventional plasma regions. Since the stable atmospheric-pressure glow (APG) discharge plasmas have been demonstrated to show two-dimensional and homogeneous broadening [1], they can be used in plasma processing as spatially uniform and high density plasma sources. Specifically, they can be applied to the surface treatment of organic materials [4], the formation of carbon-based substances such as carbon nanotubes (CNTs) [5], and sterilization [6].

On the other hand, it has recently been reported that arc discharge plasmas can be generated in liquid media in order to form new functional materials and to decompose waste gases from the viewpoint of high-efficiency processing using high density plasmas in liquid [7]. However, the plasma behavior at the boundaries between liquid and plasmas generated in liquid, i.e., the gas-liquid interface, has never been investigated, despite the fact that this interface is the most important parameter for controlling the formation of materials using plasmas.

It is therefore important to develop a novel plasma source of the atmospheric-pressure glow discharge in contact with solution for the purpose of investigating the gas (APG discharge plasma) - liquid (solution) interface in detail. Since an APG discharge plasma making contact with solution can be applied for the new functional material formation, we adopt liquid paraffin as solution. Liquid paraffin consists of hydrocarbons and is suitable for the formation of CNTs at the interface. Furthermore, when an organic molecule or DNA is included in the solution, the molecule or DNA is expected to be encapsulated into CNTs, yielding a new electronic functionality.

In this paper, we demonstrate the generation of the atmospheric-pressure glow discharge plasma in contact with liquid paraffin.

Figure 1 shows a schematic of the experimental apparatus used for generating the APG discharge plasma in contact with solution by using a capacitively coupled plasma method. The APG discharge plasma is generated by applying a radio frequency (RF) of $13.56 \mathrm{MHz}$ to a powered electrode filled with

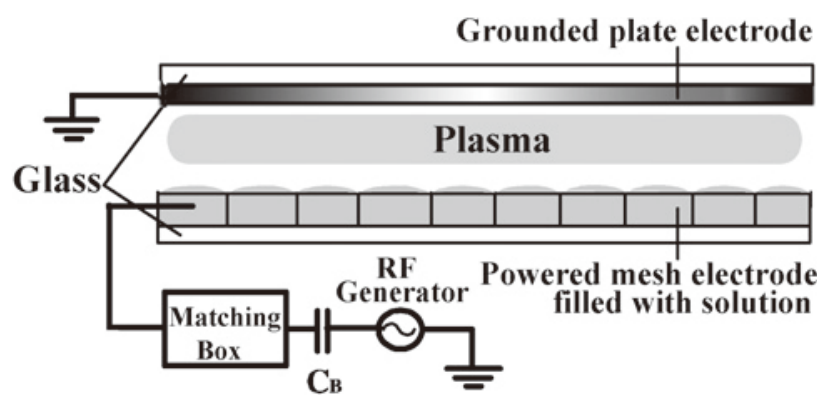

Fig.1 Schematic of experimental apparatus for atmosphericpressure discharge plasma in contact with solution by using a capacitively coupled plasma method with mesh electrode. 
solution through a matching circuit and a blocking capacitor $C_{B}$, where the RF power is within $15 \mathrm{~W}$. Since the solution that is fed into the powered electrode easily comes to touch the opposite grounded electrode due to the influence of the surface tension of the solution, a mesh electrode is used as the powered electrode to suppress the surface tension by dividing the solution into separate small cells. In addition, the mesh electrode is effective for generating the APG discharge plasma due to the concentration of the electric field on the wires of the mesh. The mesh used is made up of $0.35 \mathrm{~mm}$ diameter stainless (SUS) wires and has a fineness of 20 meshes/inch. A helium gas is adopted to generate the APG discharge plasma [8] in contact with solution. The electrode gap is varied from 200 to $1000 \mu \mathrm{m}$. The solution is liquid paraffin or water.

The homogeneous APG discharge plasma is realized using the mesh electrode without solution. In the case that liquid paraffin is fed into the mesh electrode, the APG discharge helium plasma is also stably generated by applying an RF power of 9W, as shown in Fig. 2. It is expected that liquid paraffin that consists of hydrocarbons is available for the formation of carbon nanotubes. Here, the grounded electrode is replaced by a mesh electrode for the purpose of taking a picture. On the other hand, it appears to be difficult to generate APG discharge plasma by using water.

Figure 3 presents the dependencies of the electrode gap $L_{\text {gap }}$ on the discharge voltage $V_{\mathrm{d}}$ using the mesh electrode without solution and with liquid paraffin. It is confirmed that the discharge voltage is in proportion to the electrode gap, and the discharge is stably sustained up to $L_{\text {gap }}=1 \mathrm{~mm}$. The dependence of the electrode gap on the discharge voltage without solution is similar to that with liquid paraffin. It is found that liquid paraffin as solution does not have a serious influence on the APG discharge plasma.

In conclusion, we have succeeded in the generation of an APG discharge plasma in contact with liquid paraffin as solution by using the mesh electrode. This plasma source enables us to investigate the plasma behavior at the gas-liquid interface in detail. Furthermore, new functional materials such as DNA encapsulated CNTs are expected to be formed at the interface by the introduction of DNA into liquid paraffin.

[1] S. Kanazawa et al., J. Phys. D: Appl. Phys. 21, 838 (1988).

[2] F. Massines et al., Surface Coat. Tech. 174, 8 (2003).

[3] M. Kogoma et al., J. Phys. D: Appl. Phys. 27, 1985 (1994).

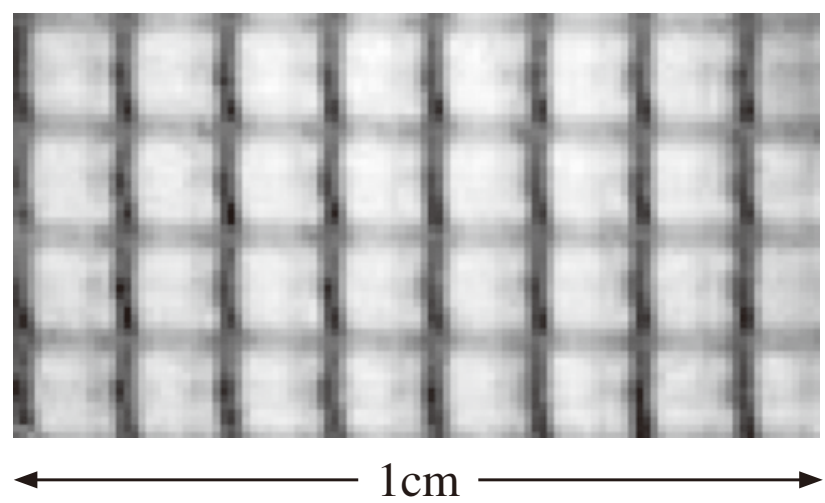

Fig. 2 Photograph of the APG discharge helium plasma generated by using the mesh electrode (SUS, 20 meshes/ inch) filled with liquid paraffin (RF power: $9 \mathrm{~W}$ ).

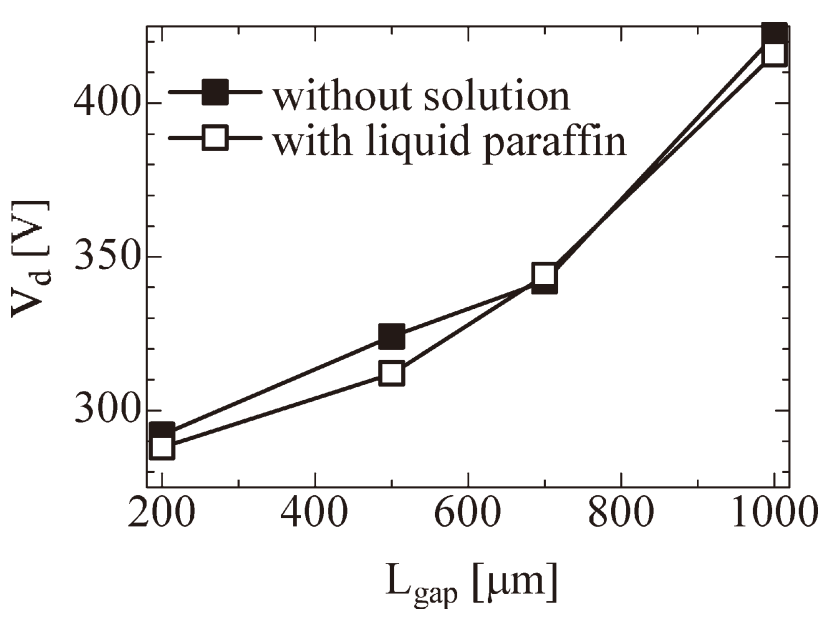

Fig. 3 Dependencies of the electrode gap $L_{\text {gap }}$ on the discharge voltage $V_{d}$ using the metal mesh electrode (SUS, 20 meshes/inch) without solution and with liquid paraffin.

[4] R. Prat et al., Polymer 41, 7355 (2000).

[5] T. Nozaki et al., J. Phys. D: Appl. Phys. 35, 2779 (2002).

[6] M. Laroussi et al., IEEE Trans. Plasma Sci. 24, 1188 (1996).

[7] N. Sano et al., Nature 414, 506 (2001).

[8] H. Yoshiki et al., Thin Solid Films 407, 156 (2002). 